\title{
Effects of the probiotic Lactobacillus animalis in murine Mycobacterium avium subspecies paratuberculosis infection
}

\author{
Enusha Karunasena ${ }^{1 *}$, Paresh C Kurkure², Russell D Lackey², Kevin Wyatt McMahon ${ }^{1}$, Estevan P Kiernan², \\ Suzanne Graham ${ }^{5}$, Magdy S Alabady ${ }^{4}$, David L Campos ${ }^{3}$, Owatha L Tatum ${ }^{5}$ and Mindy M Brashears ${ }^{2}$
}

\begin{abstract}
Background: MAP is a suspected zoonotic pathogen and the causative agent of Johne's Disease in cattle and other ruminant animals. With over $\$ 1$ billion dollars in loss to the dairy industry due to Johne's Disease, efforts to eliminate or reduce MAP from cattle are of importance. The purpose of this study was to determine if daily intake of probiotics could eliminate or reduce Johne's Disease associated symptoms and pathogenesis by MAP. Post infection, animals are often asymptomatic carriers with limited shedding of the pathogen, proving early detection to be difficult. Disease and symptoms often appear 3-4 years after infection with antibiotic treatment proving ineffective. Symptoms include chronic gastrointestinal inflammation leading to severe weight-loss from poor feed and water intake cause a wasting disease. These symptoms are similar to those found in individuals with Crohn's Disease (CD); MAP has been implicated by not proven to be the causative agent of CD. Probiotics administered to livestock animals, including dairy and beef cattle have demonstrated improvements in cattle performance and health. Our objectives included determining the benefits of Lactobacillus animalis (strain name: NP-51) in MAP infected BALB/C mice by evaluating systemic and gastrointestinal response by the host and gut microbiota. Male and female animals were fed $1 \times 10^{6} \mathrm{CFU} / \mathrm{g}$ probiotics in sterile, powdered mouse chow daily and infected with $1 \times 10^{7} \mathrm{CFU} / \mathrm{ml} \mathrm{MAP}$ and compared to controls. Animals were evaluated for 180 days to assess acute and chronic stages of disease, with sample collection from animals every 45 days. MAP concentrations from liver and intestinal tissues were examined using real time-PCR methods and the expression of key inflammatory markers were measured during MAP infection (interferon-gamma [IFN- - ], Interleukin-1a, IL-12, IL-10, IL-6, and Tumor necrosis factor alpha [TNF-a]).
\end{abstract}

Results: Our results demonstrate administration of probiotics reduces production of IFN- $Y$ and IL-6 while increasing TNF- $a$ and IL-17 in chronic disease; healthful immune responses that reduce chronic inflammation associated to MAP infection.

Conclusions: We observed that the immune system's response in the presence of probiotics to MAP contributes towards host health by influencing the activity of the immune system and gut microbial populations.

Keywords: Probiotics, Mycobacterium paratuberculosis, Direct-fed microbials, Johne's disease, Microbiota, Lactobacillus, Crohn's disease, BALB/C

\footnotetext{
* Correspondence: enusha@vbi.vt.edu

'Virginia Tech, MC 0477, Washington Street, Blacksburg, VA 24061, USA

Full list of author information is available at the end of the article
} 


\section{Background}

Mycobacterium avium subspecies paratuberculosis (MAP) is a suspected zoonotic pathogen, associated with a wasting disease in ruminant animals (predominantly dairy cattle) known as Johne's Disease (JD). This disease leads to chronic gastrointestinal tract (GIT) inflammation, preventing animals from absorbing nutrients and decreased feed intake, and accompanied with severe diarrhea. Although, infection by MAP is found to occur in utero or during weaning - through milk or fecal contamination of water and feed- JD does not appear in cattle until the age of 210 years [1]. It invades the host through specialized ileal tissue called Peyer's patches and then enter macrophage. After infection, MAP survives in macrophages, within the small intestine, for years without triggering any systemic response from the immune system.

The clinical stage manifests when MAP begins to spread into lymph nodes flanking the GI tract, leading MAP to spread systemically; it is at this point that the symptoms of disease begin to appear [1-4]. Antibiotics are not effective in controlling JD once symptoms begin and the disease is ultimately fatal. The cost of JD to the cattle industry is over $\$ 1$ billion dollars within the dairy industry, due to higher rates of culled cattle, poor milk production or low quality products $[1,2]$.

\section{MAP is a suspected pathogen for crohn's disease}

Equally of significance are the symptoms of disease and pathology from MAP-associated JD which are similar to Crohn's Disease (CD) - a chronic inflammatory bowel syndrome occurring in humans. Immunocompromised patients - such as AIDS patients - are susceptible to MAP infection $[1,2,5,6]$. MAP is linked (though not confirmed) to cause CD $[1,7]$. Many CD patients harbor MAP in their GIT tissues [8]. Introduction of subclinical animals with JD to isolated communities has demonstrated an increase in the population of JD in other livestock animals followed by increases in CD in the human population [7]. Additionally, therapies used to treat JD have been found to be effective with treatment of some CD conditions, further demonstrating associations between to the two conditions $[1,7,9,10]$.

\section{MAP-induced chronic gut inflammation}

Once MAP enters macrophages, the host's immune response 'walls-off' the infection with the accumulation of mostly other macrophage, forming a circular-shaped granuloma- characteristic of infection $[1,2,10]$. MAP induces cell-mediated immune response via T-helper-1 (Th1) cells, leads to increased production of IL-1, INF- $\gamma$, IL-6, and IL-12 family cytokines which stimulate more macrophage to the site of acute-infection $[1,8,11,12]$. Though MAP cells are killed by macrophages, more cells enter into macrophages and multiply, new MAP are then able to further infiltrate the GI tract; these conditions create a cycle of continuous infection and inflammation, causing lesions to expand [1]. This is followed by infected macrophages entering neighboring lymph nodes and other organs through the vascular system, causing the spread of granulomatous inflammation. Penetration of infected tissue by multitudes of lymphocytes and macrophages leads to visible thickening of intestines, this prevents absorption of nutrients and causes diarrhea $[1,2]$. As the disease progresses, the immune response shifts from proinflammatory responses to increased production of TGF- $\beta$ and IL-10 which suppress Th1 activity $[8,11,12]$. However, IL- $1 \alpha$ is produced constitutively by macrophage at the site of infection leading to tissue scarring and damage from reactive oxygen species (ROS) $[8,11,12]$. As chronic inflammation persists, an increase in IL-10 and IL-2 production follows $[8,11,12]$.

\section{Direct-Fed microbials reduce gut inflammation}

More recently, with the use of direct-fed microbials (DFM; probiotics) in dairy cattle producers have observed decreased rates of culled cattle and animal morbidity, through wasting. The use of probiotics in the food industry is becoming an increasingly important component to developing safer and healthier foods for the public. Probiotics are organisms that are found to contribute to systemic and gut health [13-16]. Traditionally, these organisms are classified as lactic acid bacteria (LAB) that are used to ferment foods like cheese, yogurt, wine, and meat products [15]. However, their use in the medical, agricultural and scientific community is evolving [14-19]. Probiotics used in commercial foods are mostly Lactobacillus sp. and Bifidobacterium sp. [18,20-22]. The use of these organisms offers many advantages, such as bacteriocins $[14,17,19,22]$. Bacteriocins are peptides or proteins that have antibiotic properties $[14,17,19,22]$. In addition, probiotics produce other protective compounds, like hydrogen peroxide, benzoic acid, lactic acid, and biogenic amines (from the decarboxylation of amines), which decrease food-borne pathogen viability $[13,18,19]$. Also, tumor suppression studies in murine breast cancer models have demonstrated that fermented milk products by Lactobacillus sp. are able to diminish the size of tumor growth and induce increased production of antitumor immune responses $[14,23,24]$. These studies reveal reductions in inflammatory-mediated diseases by beneficial microbes found in food products. Studies conducted by M.M. Brashears and associates have demonstrated health benefits and improved performance by cattle fed NP51; NP-51 has been demonstrated to reduce Escherichia coli $\mathrm{O} 157$ and Salmonella species shedding $[16,25]$. Currently, NP-51 is used by the dairy and beef industries as a direct-fed microbial. For these reasons, we decided to use NP-51 as a DFM in this study. 
Our hypothesis for this study is that probiotics will contribute towards the reduction or elimination of chronic inflammation associated with symptoms of Johne's Disease that are produced by MAP. Our objective was to evaluate animal health in an in vivo murine model infected with MAP and fed probiotics (Lactobacillus animalis; NP-51), and to further assess changes in the following: MAP concentrations in target tissues (liver and intestinal), pathology, the expression of key inflammatory markers, and changes to the gut microbiota.

\section{Results \& discussion}

\section{MAP concentrations in intestinal and liver tissues}

Data described in Table 1 and Figure 1 and Figure 2 reveal that MAP cells were present in intestinal tissues and the liver- organs which are associated with MAP infection and pathogenesis. Additionally, these data demonstrate that regardless of NP-51 consumption viable MAP cells were able to invade host tissues- as evidenced by granuloma formation in liver samples of animals fed viable or nonviable NP-51. However, lower concentrations of MAP cells were observed in both intestinal and liver tissues at Day 90 (45 days post MAP infection) in animals that were also fed viable or nonviable NP-51, although not significant. There were no significant changes in MAP concentrations from intestinal tissues and an increase in liver MAP concentrations were observed from Day 90 through Day 180, suggesting that MAP viability may not be deterred through the presence of probiotics (see Figure 1 and Figure 2).

\section{Host immune response to MAP}

\section{Cytokine production by the host to MAP versus MAP with} NP-51 (probiotic)

Cytokines measured through a multi-plex antigen capture assay (Mouse Cytokine 20-Plex Panel; Invitrogen) or transcript expression assays were focused towards

Table 1 Total animals $(n=4)$ demonstrating granuloma formations in liver tissues

\begin{tabular}{lllll}
\hline & K-MAP & K-MAP + L-NP-51 & L-MAP & L-MAP + L-NP-51 \\
\hline Day 90 & $3 / 4$ & $3 / 4$ & $4 / 4$ & $4 / 4$ \\
Day 135 & $2 / 2^{*}$ & $3 / 4$ & $4 / 4$ & $3 / 4$ \\
Day 180 & $3 / 4$ & $2 / 4$ & $2 / 4$ & $3 / 4$ \\
\hline
\end{tabular}

Tissues were stained with Hemotoxylin \& Eosin ( $\mathrm{H} \& \mathrm{E}$ stain) prior to evaluation. For K-MAP samples at Day 135, only two sets of animal tissues were available for examination due to early expiration of animals before the harvest date (these data are highlighted with $\left.{ }^{\left({ }^{*}\right.}\right)$. Control animals did not demonstrate granuloma formation at Day 90 and Day 180; Day 135 control animals were contaminated and were positive for granulomas in liver tissues. The data represent the number of animals that demonstrated granuloma formations per total animals examined ( =4). Experimental groups included are the following: animals fed normal chow and infected with viable MAP cells (Live-MAP; L-MAP); animals fed viable probiotics in chow and uninfected (Live NP-51; L-NP-51); animals fed viable probiotics in chow and infected with non-viable MAP cells (K-MAP + L-NP-51); animals fed viable probiotics in chow and infected with viable MAP cells (L-MAP + L-NP-51). These data demonstrate MAP infection of tissues regardless of viable or non-viable NP-51 consumption. Additionally, these data evidence that host tissues produce granulomas from exposure to K-MAP antigens. inflammatory markers associated with MAP infection, including interferon-gamma (IFN- $\Upsilon$ ), TNF- $\alpha$, IL-1 $\alpha$, IL-12, IL-6, and IL-17. To determine changes in cellular activity within tissues due to viable or non-viable MAP and the introduction of NP-51 we preformed assays to measure host transcript expression for key inflammatory markers. Host immune cells may produce and store non-specific, pro-inflammatory cytokines in the event of infection and yield more specific cytokines as disease progresses. For these reasons, our evaluation of cytokine transcript concentrations was to determine their active production, post MAP infection. These results are highlighted in Figures 3 and 4, respectively.

With viable MAP (L-MAP) infection, the immune response produced is characteristic of Th1 cell responses to intracellular pathogens with the production of IFN- $\Upsilon$, IL-6, IL-12 (as described in Figure 3) [1,2,8]. In animals that were infected with viable MAP and fed viable probiotics (L- MAP + L-NP-51) - there is IFN- $\Upsilon$ production likely due to intracellular infection by MAP but this response is weaker compared to animals infected only with viable MAP, (see Figure 3). Equally, IL-12 levels are elevated but with NP-51 consumption we again observe a decrease in IL-6 circulation and an increase in proinflammatory cytokine- TNF- $\alpha$. However, with viableMAP infection, IL-17 circulation decreases.

IL-17 is a member of the IL-12 family; as IL-12 production increases Th17 cells are activated, producing a more selective, pathogen-associated immune response $[23,24,26,27]$. Our data demonstrate that animals infected with viable MAP have higher levels of IL-17 transcript expression compared to all other experimental groups (see, Figure 4). In animals infected with viable MAP and fed viable probiotics there is decreased suppression of IL-17, although IL-12 decreases. This compared to animals injected with nonviable MAP or animals fed L-NP-51 alone, further demonstrating that NP-51 is contributing towards a beneficial immune response in the host against viable MAP. Additionally, animals injected with nonviable MAP (K-MAP) and fed L-NP-51 demonstrated IL-17 expression, possibly due to increased IL-12 activity. As IL-12 circulation decreased, IL-17 also decreased. Furthermore, in the presence of NP-51 the host is able to increase TNF- $\alpha$ production, a pro-inflammatory response that normally decreases in chronic MAP infections to evade host immune activity. This increase in TNF- $\alpha$ circulation in animals fed L-NP-51 and infected with L-MAP or injected with K-MAP correlates with a decrease in IL-6 a cytokine that contributes to tissue damage in chronic inflammatory diseases, including MAP [20-23]. These results are described further in Figures 3 and 4.

Distinguishing immune responses to viable versus nonviable MAP demonstrates unique cytokine profiles 




for K-MAP (but absent for L-MAP). Animals injected with nonviable MAP show increased expression of IL-12 and IL- $1 \alpha$; however, without intracellular pathogenesis IFN- $\Upsilon$ and IL-6 were not present (see Figure 3). However, in animals that were injected with nonviable MAP and fed viable probiotics (K-MAP + L-NP-51), IFN- $\Upsilon$ remained low, likely because there is no intracellular infection. Yet, there is IL-12 production with K-MAP, possibly due to immune responses produced against circulating MAP antigens (Figure 3).
Host immune response to probiotic (NP-51)

Similar to previous studies on probiotic strains of Lactobacilli, these data (see Figure 3) suggest that NP-51 contributes to host regulation of immune response by shifting reactions toward homeostasis by increasing or decreasing pro and anti-inflammatory pathways [16-22].

Unlike animals that received K-MAP only, those injected with K-MAP and fed L-NP-51 had increased circulation of IL-17 and TNF- $\alpha$ with decreased production of IL-6 (see Figure 3). In the presence of K- 




MAP, NP-51 increased pro-inflammatory responses (higher expression of TNF- $\alpha$ and IL-17) and inhibit IL-6; IL-6 causes chronic inflammatory damage during MAP infections $[1,2,11]$. Animals injected with K-MAP demonstrate a decrease in transcript production for all cytokines relative to controls (Figure 4). However, with L-MAP there is an increase in IFN- $\Upsilon$, IL-17, IL-6, TNF- $\alpha$, and decreased gene suppression of IL-12. With L-NP-51, further gene suppression for all cytokines relative to control and all other experimental groups are reported (see
Figure 4). With animals injected with K-MAP and fed L-NP-51, there is decreased suppression of IL-6, TNF- $\alpha$, and IL-17 compared to animals fed NP-51 alone; this may be due to the presence of K-MAP antigen inducing chronic inflammatory markers. In animals infected with L-MAP and fed NP-51 (similar to K-MAP + L-NP-51) there is decreased suppression of gene transcription for IL-17, IL-6, and TNF- $\alpha$; additionally, compared to L-MAP alone, L-MAP + L-NP-51 animals have decreased IL-6 production. 


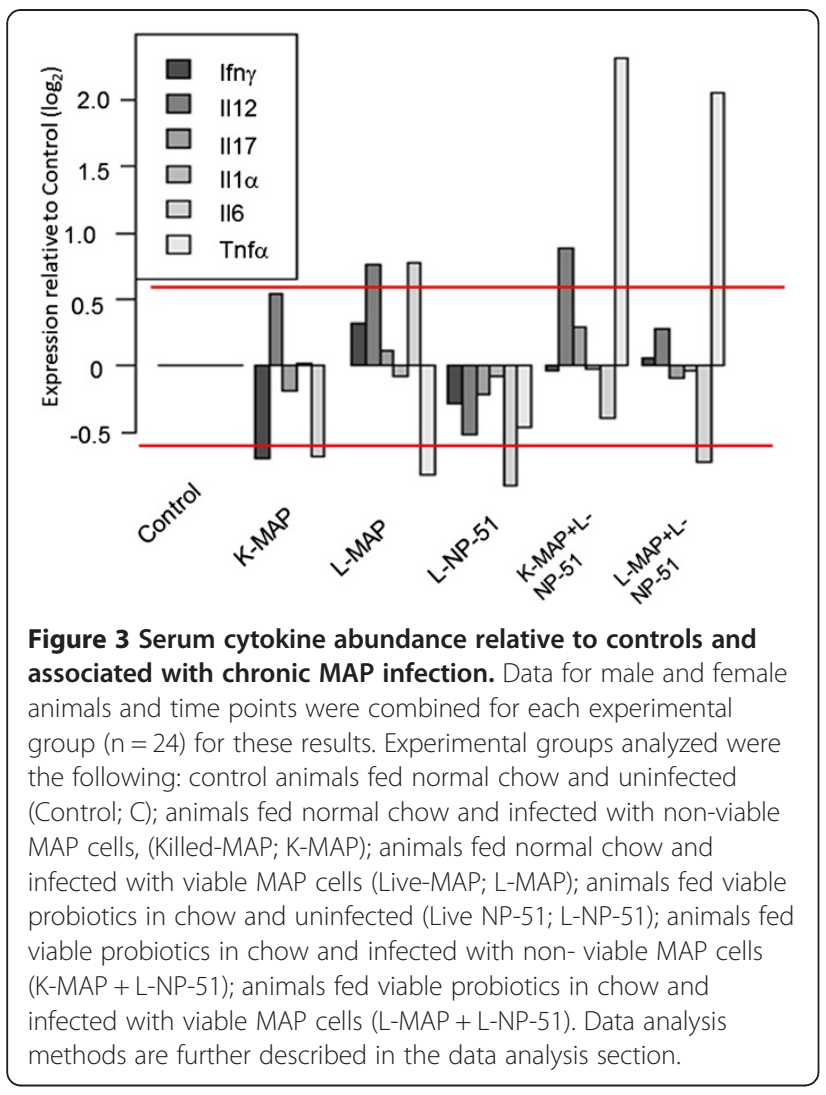

It is known that concentrations of circulating cytokines and their transcript levels are not strongly correlated, suggesting that immune cells produce and store early response cytokines and chemokines, such as TNF$\alpha$, IL-1, and INF- $\Upsilon$. However, as a pathogen persists the host begins to transcribe more specific cytokines, such as IL-17, IL-6, or IL-12, in addition to early response cytokines [9,24,26-29]. Our studies demonstrate that the administration of NP-51 alone down-regulates all of the studied cytokines, relative to control (Figure 4). There is an increase in TNF- $\alpha$ transcript expression in animals fed LNP-51 that were also infected with L-MAP or injected with K-MAP; these results are similar to serum-cytokine results (see Figure 3 and 4). This further highlights the contributive role of NP-51 in host pro-inflammatory responses, in animals with MAP. Additionally, with animals fed L-NP-51 and infected with L-MAP there is increased repression of IL-6 transcript production compared to L-MAP infected animals- further demonstrating beneficial immune responses by NP-51 in chronic MAP associated inflammation. Comparable to serum cytokine results, transcript expression by animals fed L-NP-51 and infected or injected with L-MAP or K-MAP demonstrate a shift towards homeostasis in immune activity by producing pro and anti-inflammatory responses. These data are presented in Figures 3 and 4.

\section{Associations between immune response and gut microbiota}

With chronic gut inflammatory diseases the gut microbiota - in addition to host immune responses - contributes towards disease and health [17,19,24,26-29]. Our results (described in Figure 5) demonstrate a positive correlation between gut microbiota and host immune responses, which can be either beneficial or harmful. With MAP infection, increases in INF- $\Upsilon$ and IL- 6 can lead to tissue damage [1,2,8-12,24,26-32]. Additionally, shifts in gut flora can contribute to these immune responses [17,19,24,26-29]. Studies have demonstrated that human patients with irritable bowel syndrome (IBS) or colitis experience shifts in gut flora to higher concentrations of some species of Bacterioidetes which are associated with enhanced IL-12 or IFN- $\Upsilon$ production, or increases in Proteobacteria and decreases in Firmicutes due to increases in IL-6 [17,19,24,26-29]. Enhanced growth of Actinobacteria are also associated with dysbiosis of the gut in multiple diseases- including obesity, Type I diabetes, IBS, or Crohn's disease [33]. MAP belongs to the phylum Actinobacteria [1]. Additionally, with individuals who have IBS amplified IL-17 production is found to promote healthy Firmicutes $[24,26,28]$. Similar to these studies, our data demonstrate greater populations of organisms belonging to the phylum Bacterioidetes associated with INF-Y, and nearly all organisms associated with Proteobacteria correlating with IL-6 (see Figure 5). Thus, comparing the immune responses of our experimental groups with these data, we observe higher concentrations of INF- $\Upsilon$ and IL-6 in animals infected with viable MAP when compared to experimental groups fed NP-51 (L-MAP + L-NP-51 and K- MAP + L-NP-51)therefore, animals with L-MAP demonstrate less beneficial flora and immune responses compared to groups fed probiotics (NP-51). Therefore, it is more likely that animals with L-MAP would support less beneficial immune responses and gut flora. Actinobacteria populations are also found to group with IL-6 production and some with INF- $\Upsilon$ production or IL- $1 \alpha$ down-regulation $[24,26,28]$. As such, with our cytokine expression data (Figure 3) we see higher concentrations of IL- 6 and INF- $\Upsilon$ expression in experimental groups with viable MAP (L-MAP) infections, when we compared these data to our gut floraActinobacteria correlate with the expression of IL- 6 and INF-Y; a less beneficial outcome for the host.

More so, Lactobacillus species, and predominately organisms belonging to the phylum Firmicutes, are associated with IL-17 production, especially when bacterocins are produced - suggesting a healthy hostmicrobe association with Th17 cell response and Lactobacilli $[24,27]$. This suggests the production of IL-17 and reduction in IL-6 and INF- $\Upsilon$ expression in host tissue when NP-51 is present may reduce the proliferation of 


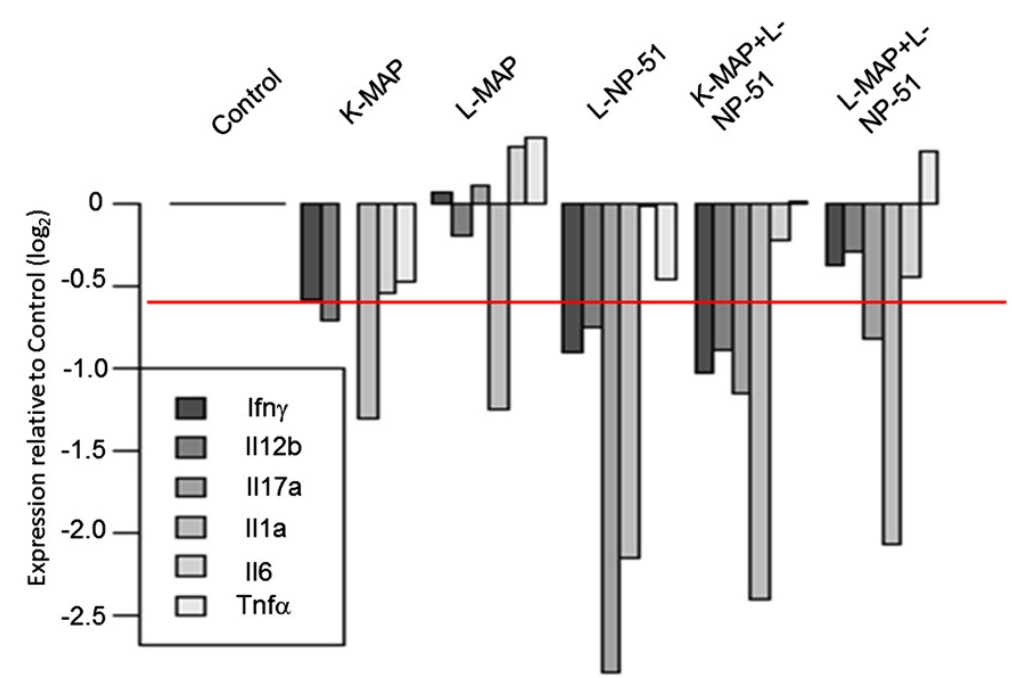

Figure 4 Tissue cytokine transcript abundance relative to controls and associated with chronic MAP infection. Data for male and female animals, time points, and tissues (small/large intestine and liver) were combined for each experimental group $(n=24)$. Experimental groups analyzed were the following: animal fed normal chow and uninfected (Control; C); animals fed normal chow and infected with non-viable MAP cells, (Killed-MAP; K-MAP); animals fed normal chow and infected with viable MAP cells (Live-MAP; L-MAP); animals fed viable probiotics in chow and uninfected (Live NP-51; L-NP-51); animals fed viable probiotics in chow and infected with non-viable MAP cells ( K-MAP + L-NP-51); animals fed viable probiotics in chow and infected with viable MAP cells (L-MAP + L-NP-51). Data analysis methods are further described in the data analysis section.

Proteobacteria and Bacterioidetes organisms that otherwise contribute to chronic gut inflammation.

Our data demonstrate NP-51 to be a beneficial gut microbe which adds to systemic host health by promoting healthful microbes in the intestinal tract that produce immune responses necessary towards homeostasis of the gut and immune system- reactions essential for reducing MAP associated disease and pathogenesis.

Probiotics have a variety of contributive effects, including the regulation of inflammation and the composition of extracellular flora in the lumen of the intestine. Through our results we were able to observe such changes in the host through the addition of NP-51 to rodent diets. However, benefits towards reducing MAP- an intracellular pathogen- were less evident in this study. These results may complement other areas of probiotic research which demonstrate reductions in MAP through the use of "unconventional bacteria"- meaning probiotics able to specifically effect intracellular pathogens [34-36]. Recent studies conducted by Click et al., show reductions in MAP concentrations in dairy cattle through the use Detzia subspecies (C79793-74) [37]. This organism is able to reduce MAP concentrations in in utero infected animals compared to most probiotics which effect extracellular loads [37]. Most studies on MAP have been focused toward eliminating mucosal inflammation and ulceration. Our studies on NP-51 support a variety of effects that appear to control this secondary inflammation. As such, this further reinforces ideas of combining probiotic organisms with differing mechanisms of action to benefit host health. Here,
NP-51 is able to reduce gastrointestinal inflammation due to MAP infection; combining NP-51 with other successful probiotics that trigger reductions in pathogen proliferation could increase these benefits.

\section{Conclusions}

There is compounding evidence to suggest that diseases due to chronic inflammation- including $\mathrm{CD}$, autoimmune disorders, and asthma- share similar mechanisms of cell-mediated immune responses [9,23,30-32]. Several studies have shown that having symptoms of chronic inflammation: tissue swelling, high immune cell responsiveness, production of ROS contribute to increased oxidative stress - leading to harmful effects in host tissues [3032]. As the incidence of inflammatory diseases (like asthma, atherosclerosis, diabetes, IBS and obesity) increase in Western nations, some groups have shown the early use of antibiotics can change the composition of microorganisms in the gut, causing increased T-cell mediated responses in airways that then cause asthma [27]. Some of these factors that increase during gut inflammation- like IL-6, IL-23 (IL-12 family cytokine), and transcription factor NF- $\mathrm{kB}$ - are also found to activate cancerous behavior $[31,38]$. Research demonstrates the accumulation of IL-6 in colon tumors causes' growth and increase in tumor size [31]. The mechanisms by which beneficial microbes contribute to host health are a multifaceted and integrated system; these studies support our research and the importance of the host, their flora, and the impact of exchange by the two, to overall health. 




These data further support studies that demonstrate the influence of probiotics in regulating chronic gut inflammation, pathogens, or the colonization of foodborne microorganisms (specifically, pathogenic Proteobacteria -Salmonella, Campylobacter species and Escherichia coli) by minimizing factors in the gut otherwise conducive to the proliferation of these and other bacteria belonging to Bacterioidetes or Firmicutes. Based on this study, we further hypothesize that contributions by beneficial microbes towards maintaining gut homeostasis may reduce 
symptoms associated with systemic disease that are produced by gut pathogens and these responses may have sexspecific differences.

\section{Methods}

\section{Experimental design}

A power test was used to determine the number of animals per experimental group. The coefficient of variance ( $\mathrm{CV} \%$ ) was 5 ; the percent difference from the control was decided at 10 ; and $\mathrm{P} \leq 0.05$ with a power of $90 \%$. The number of replicates per treatment was determined to be 5 mice/experimental group. Assistance in developing the experimental design was through personal communications with M.M. Galyean (TTU, Dept. of Animal \& Food Sciences) and with reference to Martin, Meek, and Willeberg. 1987; Veterinary Epidemiology Principals and Methods; p. 45 Iowa State University Press. Ames, Iowa. In detail the study design is described in Table 2.

For this study, 23-28 day old BALB/c mice equally divided between male and female, for a total of 410 animals were tested. (Charles River Laboratories, Wilmington, MA). Animals were acclimated for 2 weeks in the Texas Tech University (TTU) Animal Care and Use (ACU) facilities prior to experimentation and animal welfare, housing conditions, and euthanasia were according to protocols established through TTU-ACU (ACUC Approval Number: 07060-12). Five animals per experimental group were housed in sterilized cages with sterilized bedding. Animals were provided with sterile water and mouse chow, ad libitum. There were a total of 10 experimental groups and four time-points over the course of 180 days, sample collections were conducted at days $45,90,135$, and 180 . At day 0 , five-male and five-female mice were euthanized and tissues were collected for histopathology and cryogenic preservation, to evaluate animals prior to experimentation. From day 0 through day 45 animals were fed a diet of: sterile powder chow, sterile powder chow combined with $1 \times 10^{6} \mathrm{CFU} / \mathrm{g}$ NP-51, or heat-killed NP-51 at similar concentrations, daily. At day 45, 100 animals from 10 experimental groups were euthanized; animals were sedated with Isoflurane inhalation, followed with cardiac puncture and blood collection. The large (colon) and small intestinal tissues, stomach, and liver from male and female animals $(n=4)$ were preserved for histopathology analysis in $10 \%$ formalin solution in phosphatebuffered saline (PBS). Identical tissues collected from male/female mice $(n=6)$ were harvested and flash frozen in liquid nitrogen, followed with long term cryogenic preservation at $-80^{\circ} \mathrm{C}$. MAP concentrations were determined, from $0.2 \mathrm{~g}$ of harvested tissues, using qRT-PCR on large intestine and liver; liver tissues presented granulomas distinct to MAP infection based on histopathology analysis.

\section{MAP cultures and cell harvesting}

MAP cultures were originally harvested from cattle at the USDA National Animal Disease Center (NADC), and kindly provided by Judith Stabel (Ames, Iowa). A single culture was shipped to TTU, in Middle Brooks H79 broth with Mycobactin (Allied Monitor, Fayette, MO), at refrigerated conditions. Cultures were grown and harvested according to conditions provided through Stabel et al., at the NADC $[39,40]$. MAP cells were rendered non-viable by boiling cultures for $20 \mathrm{~min}$ in a $65^{\circ} \mathrm{C}$ waterbath [40].

\section{NP-51 cultures and chow preparation}

Freeze-dried NP-51 with maltodextrin (MD) was shipped directly to TTU by Culture Systems Incorporated (Mishawaka, IN). Twenty-gram packets were made with NP-51, individual packets were used once daily and any remaining material was discarded. Viable cultures of NP51 were mixed into sterile, powdered mouse chow (7012 Teklad LM-485 Mouse/Rat Sterilizable Diet; Harlan Teklad Diets, Madison WI) using a KitchenAid ${ }^{\circledR}$ 5-Quart Tilt-Head Artisan Series Stand Mixer (Bed Bath \& Beyond; Lubbock, TX) at setting 2 or 3, for 15-20 minutes in a BSL-2 safety cabinet (this insured even distribution of NP51 in the powdered chow). Non-viable NP-51 was prepared by heating samples at $180^{\circ} \mathrm{C}$ in a dry oven for $20 \mathrm{~min}$ (Fisher Scientific Convection Gravity Oven; Fisher Sci, Houston, TX). Non-viable cultures were mixed with an identical mixer system, separately, using sterile bowls and utensils. Each chow was replaced daily with new feed according to experimental conditions. Animal cages and feed containers were handled under a BSL-2 safety cabinet. Feed containers were cleaned and sterilized weekly by autoclaving $\left(121^{\circ} \mathrm{C}\right.$ for $\left.15 \mathrm{~min}\right)$, new feed containers were replaced along with sterilized cages and bedding every 3rd or 7th day. Utensils for preparing chow including bowls, mixing utensils, and glassware were cleaned daily and sterilized with baking at $180^{\circ} \mathrm{C}$ in a convection gravity oven at a minimum of 4 hours or overnight, before use.

\section{MAP infection and sampling schedule}

On day 46, through intraperitoneal (IP) injection experimental groups were injected with $100 \mu \mathrm{l}$ of sterile PBS containing $1 \times 10^{7} \mathrm{CFU} / \mathrm{ml}$ viable or non-viable MAP. Controls were injected with $100 \mu \mathrm{l}$ PBS only. Animals were observed closely for $48 \mathrm{~h}$ for negative physiological reactions to IP injections. Every 45 days post infection - Days 90, 135, and 180- necropsies were performed.

\section{Serum/ tissue collection \& cytokine analysis}

At each necropsy, blood was collected into serum separation tubes, and serum was pooled from each experimental group ( $\mathrm{n}=5)\left(13 \times 100 \mathrm{~mm}, \mathrm{SST}^{\mathrm{TM}}\right.$ Serum Separation Tubes; Beckton- Dickinson; San Jose, CA). Blood samples were refrigerated for 24-48 h after collection, followed by 
Table 2 Experimental design

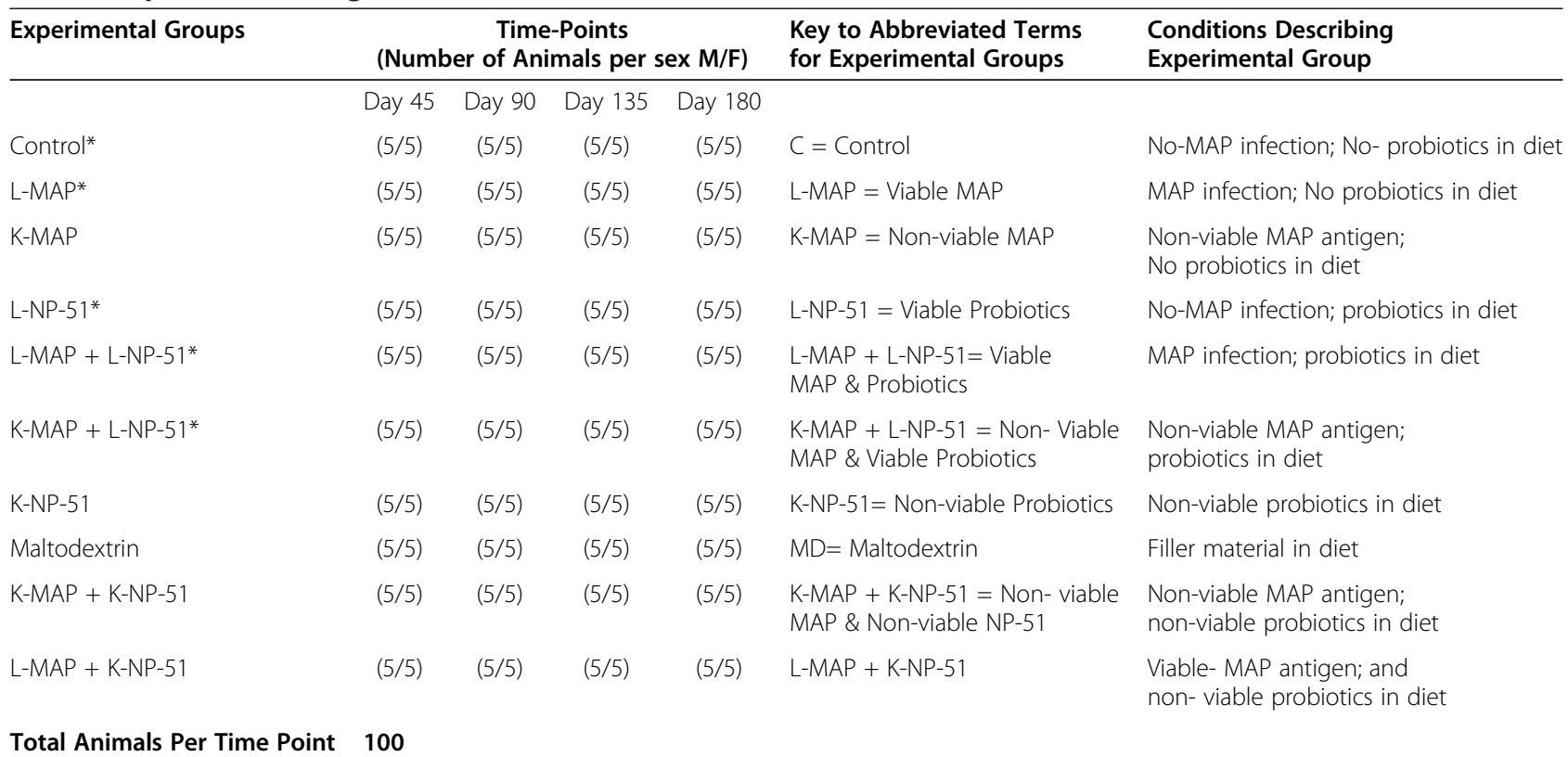

A description of each of the experimental groups, sexes, and treatments are described for one time point, each of the four time points in this study had the same experimental design. Select experimental groups were analyzed for metagenomic, transcriptomic and cytokine analysis based on histopathology results; the selected groups' are highlighted with '*'.

centrifugation at 5,000 $\times \mathrm{g}$ for $5 \mathrm{~min}$ (Marathon 2100R, Thermo-Fisher Scientific; Houston, TX). Serum was transferred, using disposable, sterile serological pipettes to sterile, $2 \mathrm{ml}$ cryogenic tubes and stored at $-20^{\circ} \mathrm{C}$ (Fisher Scientific; Houston, TX).

Two-hundred microliters of serum from each experimental condition and for all collection time points were shipped to TTUHSC, at El Paso and analyzed using a Mouse Cytokine 20-Plex Panel for the Luminex ${ }^{\circledR}$ platform - according to manufacturer protocol (Invitrogen/Life Technologies; Carlsbad, CA). Serum was analyzed in triplicate wells and compared to standards.

\section{Tissue RNA/DNA extractions, cDNA synthesis, \& cDNA analysis}

Colon tissues were ground with mortar and pestle in liquid nitrogen to preserve RNA/DNA and prevent nuclease activity in tissues. Approximately, $100 \mathrm{mg}$ of tissue were extracted for RNA using a Trizol ${ }^{\circledR}$ kit (Invitrogen, Carlsbad, CA). Co-purification of DNA from these extractions were preformed from the separated organic layer, using a DNeasy ${ }^{\circledR}$ Blood \& Tissue Kit according to protocols for total bacterial DNA extractions (Qiagen, Valencia, CA). Purified DNA were kept in 1x Tris-EDTA Buffer and concentrations were measured spectrophotometerically at a ratio of 260/280 $\mathrm{nm}$ (Nanodrop 1000, Wilmington, DE). DNA at concentrations of $40-50 \mathrm{ng} / \mu \mathrm{l}$ in $50 \mu \mathrm{l}$ of water was provided for sequencing. High throughput sequencing was conducted using $454{ }^{\circledR}$ pyrosequencing technology
(Roche Laboratories, Branford, CT) at Research and Testing Laboratories, LLC (Lubbock, TX).

Duplicate samples of RNA, collected from triplicate animals from each sex for each experimental condition were prepared for quantitative Real Time- PCR (qRT-PCR). High- Capacity ${ }^{\circledR}$ cDNA Reverse Transcription kit was used (ABI, Foster City, CA). For RNA samples with concentrations below $60 \mathrm{ng} / \mu \mathrm{l}$ a $\operatorname{High}^{\circledR}$ Capacity RNA-to-cDNA Master Mix kit was used for cDNA synthesis (ABI; Foster City, CA). cDNA were analyzed using SYBR green probes for genes of interest for Open ${ }^{\circledR}$ Array platform (Life Technologies Inc; Carlsbad, CA). Probes for all genes were selected from array panels and customized for our study- 9 plates were used in the analysis. Assays were performed by The University of Texas, Southwestern at Dallas. Analysis of data was conducted using Open ${ }^{\circledR}$ Array Real Time qPCR Analysis Software Version 1.0.4. Each cDNA sample was analyzed in duplicate, from triplicate animals and both sexes.

\section{qRT-PCR analysis of MAP concentrations from tissues}

The template DNA used for construction of standards was extracted from MAP culture. Briefly, $10 \mathrm{ml}$ of the MAP culture was pelleted using centrifugation (Marathon 2100R, Thermo-Fisher Scientific, Houston, TX) at $5000 \times \mathrm{g}$ for 15 minutes. The cells were washed twice with HPLC-grade water (Ricca Chemical Company; Arlington, TX) and again suspended in new HPLC-grade water. DNA was extracted by heating $50 \mu \mathrm{l}$ of cell suspension in PCR tubes (VWR Int, Westchester PA) at $99^{\circ} \mathrm{C}$ for 15 minutes in Gene Amp PCR 
system 2700 Thermocycler (Applied Biosystems, Foster City, CA). The heated sample was centrifuged to pellet the cell debris and the supernatant was used as template for successive experiments.

The primers used for this assay amplifies a $163 \mathrm{bp}$ region of the IS-Mav region in the MAP genome. Various primer pairs were tested before selecting the ISMav2 primers [3,4,41-43]. By using plasmids with the $163 \mathrm{bp}$ fragment DNA insertion as standards, serial dilutions were tested to develop a standard curve and then enumerate the number of MAP cells in the experimental samples by plotting the $\mathrm{Ct}$ values on the curve. This was confirmed using the melting curve analysis of the PCR product which showed only one peak for ISMav2; thus the amplicon was very specific for MAP. Using recombinant plasmids at specific concentrations as standards we tried to do an absolute quantification of MAP but based on the standard curve generated using these plasmids the minimum number of cells that can be detected was 10. PCR reactions were carried out in $50 \mu \mathrm{l}$ containing primer ISMav2 (Forward seq 5'-CGG CAA AAT CGA GCA GTT TC-3'; Reverse seq 5' ${ }^{\prime}$-TGA GCC GGT GTG ATC TTT-3'), $10 \mu \mathrm{l}$ of template DNA, using Qiagen $\operatorname{Hot}^{\circledR}$-Start PCR kit (Qiagen Sciences, MD) following manufacturer protocols [3]. The PCR products were run on $2 \%$ agarose gel stained with $\mathrm{EtBr}$ in $1 \mathrm{X}$ TAE buffer to check for a single amplicon. The PCR product was purified using Qiagen ${ }^{\circledR}$ PCR-Purification Kit (Qiagen Sciences, MD) and used for direct cloning using pGEM-T ${ }^{\circledR}$ Easy vector system (Promega Corporation, Madison, WI) in $\mathrm{HB}_{101}{ }^{\circledR}$ competent E. coli cells (Promega Corporation, Madison, WI) following manufacturer's protocol. The recombinant plasmids were purified using Quick ${ }^{\circledR}$ Plasmid mini- prep kit (Invitrogen, Carlsbad, CA) following manufacturer's methods and were sequenced at the Biotech Core Facility (Texas Tech University, Lubbock, TX). The sequence data was analyzed using BLAST to confirm its uniqueness to MAP. These recombinant plasmids were used as standards for RT-PCR.

The plasmid concentration was measured at $260 \mathrm{~nm}$ at a ratio of $260 / 280 \mathrm{~nm}$ using $\mathrm{ND}^{\circledR}-1000$ spectrophotometer in the TTU Biotech Core Facility. Based on the concentration and the length of the recombinant plasmids, the number of plasmids in the solution was calculated and dilutions of $10,100,1000$, and 10000 plasmids per microliter were prepared in $1 \mathrm{X}$ TE buffer.

These plasmid dilutions were used for constructing a standard curve for the quantification of MAP cells from mouse colon and liver tissue using RT- PCR. A $16 \mathrm{~s}$ rRNA sequence present in bacteria was used as the reference gene. The primer pair used for amplification of that sequence were universal primers (Forward $5^{\prime} \mathrm{CCA}$ TGA AGT CGG AAT CGC TAG-3'; Reverse 5'- ACT CCC ATG GTG TGA CGG-3').
PCR reactions were carried out in $25 \mu$ l using SuperScript ${ }^{\circledR}$ III Platinum Two step qRT- PCR kit with SYBR Green (Invitrogen; Carlsbad, CA). The reaction set up and the thermal cycling parameters were according to manufacturer's instructions. The 7500 Real-Time PCR system (Applied Biosystems; Foster City, CA) at the TTU, Biotech Core Facility was used for real time detection of amplified dsDNA with SYBR Green. Melting curve analysis was also performed according to the instrument protocol. The experimental samples were divided into 4, 96 well plates. Every sample was run in triplicate. Each plate had non-template controls for ISMav2 primers and universal primers; quantification standards were recombinant plasmids with ISMav2 representative of cell numbers $\left(1 \times 10^{5}, 1 \times 10^{3}, 1 \times 10^{2}\right.$, and $\left.1 \times 10^{1}\right)$, experimental samples were evaluated with ISMav2 primers or universal primers. Specific amplification of target DNA was monitored by comparing the normalized reporter signal (SYBR Green) for a threshold cycle $(\mathrm{Ct})$ and the signal obtained for controls.

\section{Data analysis \\ Real time PCR analysis}

Data were normalized and a One Way Analysis of Variance (ANOVA) was conducted; if the mean values among the treatment groups were greater than what would be expected by chance there was a significant difference ( $P$ $\leq 0.05)$. A post-hoc, all pairwise multiple comparison procedure (Tukey Test) was performed for statistical analysis of significance.

\section{Tissue cytokine transcript analysis}

Files from the Luminex ${ }^{\circledR}$ and Open ${ }^{\circledR}$ Array analyses were parsed and organized into tab-delimited files using custom perl scripts. Values across multiple days and sexes were averaged to result in one value for each of 6 experimental conditions (Control, L-MAP, K-MAP, L-NP-51, K-MAP + L-NP-51 and L-MAP + L-NP-51). Targets (cytokines or transcripts) that gave reliable results above background were included in the final analysis. All values were normalized to control values and expressed as log base 2 .

\section{Gut microbiota analysis}

For microbiota analysis, .sff files generated from 454 sequencing were demultiplexed, converted to .fastq files and resulting sequences were trimmed and mapped to $16 \mathrm{~S}$ ribosomal DNA intergenic regions to classify the origin of the sequence. The methodology associated with 454 sequencing were conducted by Research and Laboratory Testing (Lubbock, TX) according to protocols previously developed and described by Dowd et al., [44]. Sequencing data were deposited to GenBank short reads archive (SRA056455). The percent of sequences from each 
organism in each sample was normalized across all samples and final values were normalized to control and values were expressed as log base 2 of the difference between each sample and the control. A custom R script was written to perform a Pearson correlation between the relative abundance of each genus and relative abundance of each cytokine; geni with p-values of $<0.05$ in the Pearson and at least one cytokine from the Luminex ${ }^{\circledR}$ analysis were included in the final table, separated based on whether the r-value was positive (positive correlation) or negative (negative correlation).

\section{Abbreviations}

MAP: Mycobacterium avium subspecies paratuberculosis L-MAP- Live MAP (viable cells); K-MAP: Killed MAP (non-viable cells) L-NP51- live NP51 (viable NP51 cells) K-NP51- killed NP51 (non-viable cells); F: Female; M: Male.

\section{Competing interests}

The authors declare that they have no competing interests.

\section{Authors' contributions}

EK: Designed, coordinated and conducted the animal studies, histopathology, molecular/microbiology and immunology assays, analysis and interpretation of data/results and drafted the manuscript. PCK: Tissue collections, DNA/RNA extractions from tissues, qRT-PCR assays to quantitate MAP from intestinal tissues, and drafted a section of the manuscript on RTPCR analysis of MAP. RDL: Conducted animals feeding regimen, tissue collections, DNA/RNA extractions from tissues. KWM: Contributed to the design of qRT-PCR assays, tissue collection procedures, RNA/DNA extractions, and conducted the analyses of data for immune and microbiota assays; additionally, he drafted a section on methods for data analysis. EPK: Conducted animals feeding regimen, tissue collections, and immune cell analysis through Giemsa staining. SG: Conducted and interpreted histopathology for all animals tissues examined. MSA: Conducted the analysis of microbiota data collected through high-through put next generation sequencing methods. DC: Conducted qRT-PCR assays on liver tissues to quantitate MAP OLT: Contributed in the coordination and conduction of PCR, qRT-PCR assays on MAP. MMB: Contributed in the design and coordination of NP-51/probiotic use in the animal model, methods for probiotics intake, microbiology analysis of probiotics/MAP. All authors read and approved the final manuscript.

\section{Acknowledgements}

We would like to thank Nutrition Physiology Incorporated (NPC) and the Centers of Excellence support for the International Center for Food Industry Excellence for their contributions towards this study, including Dr. Doug Ware from NPC. We would also like to thank the TTU Core Facility and TTU Molecular Pathology Program for their assistance and contributions. Additionally, the authors would like to thank the TTU/HHMI Undergraduate Research Program for their support of David Campos. We would like to thank Dr. Judith Stabel at the NADC and Drs. Mohamed Osman and Don Beitz at ISU for their contributions.

\section{Funding}

Nutrition Physiology Incorporated provided funding for this study, including some salary for Mindy M. Brashears, Enusha Karunasena, Estevan Kiernan, Russell Lackey, and Paresh Kurkure.

\section{Author details}

${ }^{1}$ Virginia Tech, MC 0477, Washington Street, Blacksburg, VA 24061, USA. ${ }^{2} E S B$ Canton \& Main St, MS3132, TTU, Lubbock, TX 79409, USA. ${ }^{3}$ TTU/HHMI Undergraduate Research Program, Lubbock, TX 79409, USA. ${ }^{4} \mathrm{U}$ of Illinois Urbana-Champaign 1206, West Gregory Street, Urbana, IL 61801, USA. ${ }^{5}$ TTUHSC, 36014th Street, Lubbock, TX 79409, USA.

Received: 21 August 2012 Accepted: 7 January 2013

Published: 16 January 2013

\section{References}

1. Nacy C, Buckley M: MYCOBACTERIUM AVIUM PARATUBERCULOSIS:Infrequent Human Pathogen or Public Health threat? AMERICAN ACADEMY OF MICROBIOLOGY. Washington D. C: American Academy of Microbiology; 2008:1-41. www.asm.org

2. Harris NB, Barletta RG: Mycobacterium avium subsp. Paratuberculosis in veterinary medicine. Clin Microbiol Rev 2001, 14(3):489-512.

3. Schönenbrücher $H$, Abdulmawjood A, Failing K, Bülte M: New triplex real-time PCR assay for detection of Mycobacterium avium subsp. paratuberculosis in bovine feces. Appl Environ Microbiol 2008 74(9):2751-2758.

4. Slana I, Kralik P, Kralova A, Pavlik I: On-farm spread of mycobacterium avium subsp. Paratuberculosis in raw milk studied by IS900 and F57 competitive real time quantitative PCR and culture examination. Int J Food Microbiol 2008, 128(2):250-257.

5. Richter E, Wessling J, Lugering N, Domschke W, Rusch-Gerdes S: Mycobacterium avium subsp. paratuberculosis infection in a patient with HIV, Germany. Emerg Infect Dis 2002, 8(7):729-731.

6. Radomski N, Thibault VC, Karoui C, de Cruz K, Cochard T, Gutierrez C, Supply $P$, Biet $F$, Boschiroli ML: Determination of genotypic diversity of mycobacterium avium subspecies from human and animal origins by mycobacterial interspersed repetitive-unit-variable-number tandemrepeat and IS1311 restriction fragment length polymorphism typing methods. J Clin Microbiol 2010, 48(4):1026-1034.

7. Hermon-Taylor J: Mycobacterium avium subspecies paratuberculosis, crohn's disease and the doomsday scenario. Gut Pathog 2009, 1(1):15.

8. Pierce ES: Ulcerative colitis and crohn's disease: is mycobacterium avium subspecies paratuberculosis the common villain? Gut Pathog 2010, 2(1):21.

9. Lidar M, Langevitz $P$, Shoenfeld $Y$ : The role of infection in inflammatory bowel disease: initiation, exacerbation and protection. Isr Med Assoc J 2009, 11(9):558-563.

10. Sartor RB: Does Mycobacterium avium subspecies paratuberculosis cause crohn's disease? Gut 2005, 54(7):896-898.

11. Woo SR, Czuprynski CJ: Tactics of Mycobacterium avium subsp. paratuberculosis for intracellular survival in mononuclear phagocytes. J Vet Sci 2008, 9(1):1-8.

12. Abubakar I, Myhill D, Aliyu SH, Hunter PR: Detection of Mycobacterium avium subspecies paratuberculosis from patients with crohn's disease using nucleic acid-based techniques: a systematic review and metaanalysis. Inflamm Bowel Dis 2008, 14(3):401-410.

13. Macfarlane GT, Cummings JH: Probiotics and prebiotics: can regulating the activities of intestinal bacteria benefit health? BMJ 1999 318(7189):999-1003

14. Furrie E, Senok AC, Frank DN, Sullivan KE: Pondering probiotics. Clin Immunol 2006, 121(1):19-22.

15. Heller KJ: Probiotic bacteria in fermented foods: product characteristics and starter organisms. Am J Clin Nutr 2001, 73(2 Suppl):374S-379S.

16. Elam NA, Gleghorn JF, Rivera JD, Galyean ML, Defoor PJ, Brashears MM Younts-Dahl SM: Effects of live cultures of lactobacillus acidophilus (strains NP45 and NP51) and propionibacterium freudenreichii on performance, carcass, and intestinal characteristics, and Escherichia coli strain 0157 shedding of finishing beef steers. J Anim Sci 2003 81(11):2686-2698.

17. Sonnenburg J, Chen CT, Gordon Jl: Genomic and metabolic studies of the impact of probiotics on a model gut symbiont and host. PLOS BiO 2006, 4(12):e413.

18. Meier RF: Probiotics: a new treatment for antibiotic-associated diarrhea? Digestion 2005, 72(1):49-50.

19. O' Flaherty S, Klaenhammer TR: The role and potential of probiotic bacteria in the gut, and the communication between gut microflora and gut/host. Int Dairy J 2010, 20:262-268.

20. Leblanc J, Fliss I, Matar C: Induction of a humoral immune response following an escherichia coli 0157:H7 infection with an immunomodulatory peptidic fraction derived from lactobacillus helveticus-fermented milk. Clin Diagn Lab Immunol 2004, 11(6):1171-1181.

21. Minervini F, Algaron F, Rizzello CG, Fox PF, Monnet V, Gobbetti M: Angiotensin l-converting- enzyme-inhibitory and antibacterial peptides from lactobacillus helveticus PR4 proteinase- hydrolyzed caseins of milk from six species. App/ Environ Microbiol 2003, 69(9):5297-5305. 
22. Balasubramanyam BV, Varadaraj MC: Cultural conditions for the production of bacteriocin by a native isolate of lactobacillus delbruecki ssp. Bulgaricus CFR 2028 in milk medium. J Appl Microbiol 1998, 84(1):97-102.

23. Shida K, Suzuki T, Kiyoshima-Shibata J, Shimada S, Nanno M: Essential roles of monocytes in stimulating human peripheral blood mononuclear cells with Lactobacillus casei to produce cytokines and augment natural killer cell activity. Clin Vaccine Immunol 2006, 13(9):997-1003.

24. Spor A, Koren O, Ley R: Unravelling the effects of the environment and host genotype on the gut microbiome. Nat Rev Microbiol 2011, 9(4):279-290.

25. Vasconcelos JT, Elam NA, Brashears MM, Galyean ML: Effects of increasing dose of live cultures of Lactobacillus acidophilus (strain NP 51) combined with a single dose of propionibacterium freudenreichii (strain NP 24) on performance and carcass characteristics of finishing beef steers. J Anim SCi 2008, 86(3):756-762

26. Wexler HM: Bacteroides: the good, the bad, and the nitty-gritty. Clin Microbiol Rev 2007, 20(4):593-621.

27. Carroll IM, Threadgill DW, Threadgill DS: The gastrointestinal microbiome: a malleable, third genome of mammals. Mamm Genome 2009, 20(7):395-403

28. Barnes MJ, Powrie F: Immunology. The gut's clostridium cocktail. Science 2011, 331(6015):289-290.

29. Fischbach MA, Sonnenburg JL: Eating for two: how metabolism establishes interspecies interactions in the gut. Cell Host Microbe 2011, 10(4):336-347.

30. Jones $\mathrm{L}$, Loftus EV: Lymphoma risk in inflammatory bowel disease: is it the disease or its treatment? Inflamm Bowel Dis 2007, 13(10):1299-1307.

31. Fantini MC, Pallone F: Cytokines: from gut inflammation to colorectal cancer. Curr Drug Targets 2008, 9(5):375-380.

32. Baumgart DC, Sandborn WJ: Inflammatory bowel disease: clinical aspects and established and evolving therapies. Lancet 2007, 369(9573):1641-1657.

33. Shenoy AR, Sivakumar K, Krupa A, Srinivasan N, Visweswariah SS: A survey of nucleotide cyclases in actinobacteria: unique domain organization and expansion of the class III cyclase family in mycobacterium tuberculosis. Comp Funct Genomics 2004, 5(1):17-38.

34. Click RE, Van Kampen CL: Short communication: progression of Johne's disease curtailed by a probiotic. J Dairy Sci 2009, 92(10):4846-4851.

35. Click RE, Van Kampen CL: Comparison of ante-mortem assays to assess progression/regression of paratuberculosis in individual dairy animals. Virulence 2010, 1(3):134-144.

36. Click RE, Van Kampen CL: Assessment of dietzia subsp. C79793-74 For treatment of cattle with evidence of paratuberculosis. Virulence 2010, 1(3):145-155.

37. Click RE: A 60-day probiotic protocol with Dietzia subsp. C79793-74 prevents development of Johne's disease parameters after in utero and/ or neonatal MAP infection. Virulence 2011, 2(4):337-347.

38. Zisman TL, Rubin DT: Colorectal cancer and dysplasia in inflammatory bowel disease. World J Gastroenterol 2008, 14(17):2662-2669.

39. Stabel JR, Ackermann MR: Temporal Mycobacterium paratuberculosis infection in T-cell receptor (TCR)-alpha and TCR-delta-deficient mice. Vet Immunol Immunopathol 2002, 89(3-4):127-132.

40. Waters WR, Miller JM, Palmer MV, Stabel JR, Jones DE, Koistinen KA, Steadham EM, Hamilton MJ, Davis WC, Bannantine JP: Early induction of humoral and cellular immune responses during experimental mycobacterium avium subsp. Paratuberculosis infection of calves. Infect Immun 2003, 71(9):5130-5138.

41. Herthnek D, Bölske G: New PCR systems to confirm real-time PCR detection of Mycobacterium avium subsp. paratuberculosis. BMC Microbiol 2006, 6:87.

42. O'Mahony J, Hill C: Rapid real-time PCR assay for detection and quantitation of Mycobacterium avium subsp. paratuberculosis DNA in artificially contaminated milk. Appl Environ Microbiol 2004, 70(8):4561-4568.
43. Ravva SV, Stanker LH: Real-time quantitative PCR detection of mycobacterium avium subsp. Paratuberculosis and differentiation from other mycobacteria using SYBR green and TaqMan assays. J Microbiol Methods 2005, 63(3):305-317.

44. Dowd SE, Callaway TR, Wolcott RD, Sun Y, McKeehan T, Hagevoort RG, Edrington TS: Evaluation of the bacterial diversity in the feces of cattle using $16 \mathrm{~S}$ rDNA bacterial tag-encoded FLX amplicon pyrosequencing (bTEFAP). BMC Microbiol 2008, 8:125.

doi:10.1186/1471-2180-13-8

Cite this article as: Karunasena et al:: Effects of the probiotic Lactobacillus animalis in murine Mycobacterium avium subspecies paratuberculosis infection. BMC Microbiology 2013 13:8.

\section{Submit your next manuscript to BioMed Central and take full advantage of:}

- Convenient online submission

- Thorough peer review

- No space constraints or color figure charges

- Immediate publication on acceptance

- Inclusion in PubMed, CAS, Scopus and Google Scholar

- Research which is freely available for redistribution

Submit your manuscript at www.biomedcentral.com/submit
C Biomed Central 